\title{
6-Oxyindan-1-ones with dipeptide chains
}

\author{
Serhiy V. Shilin, ${ }^{\mathrm{a}^{*}}$ Olga V. Shablykina, ${ }^{\mathrm{a}, \mathrm{b}}$ Igor V. Levkov, ${ }^{\mathrm{a}}$ \\ Oleksandra I. Bugera, ${ }^{\text {a }}$ Zoia V. Voitenko ${ }^{\text {a }}$ \\ ${ }^{a}$ Department of Chemistry, Taras Shevchenko National University of Kyiv, \\ Volodymyrska Street, 64/13, Kyiv 01601, Ukraine \\ ${ }^{\mathrm{b}}$ V.P. Kukhar Institute of Bioorganic Chemistry and Petrochemistry of the NAS of Ukraine, \\ Murmanska st., 1, Kyiv, 02660, Ukraine \\ varang77@ukr.net
}

Keywords: 6-hydroxyindan-1-one, dipeptide derivatives, oxyacetic linker, activated ester method, N-hydroxysuccinimide.

Through $N$-acylation of $\alpha$ - or $\beta$-amino acid units by 2-(3-oxo-2,3-dihydro-1H-inden-5-yloxy)acetic acid using the method of $N$-hydroxysuccinimide esters new dipeptide indan-1-one derivatives were obtained. In general, the direct interaction of the acetic carboxyl group of the substrate with the amino group of the $\alpha$ - or $\beta$-dipeptide is a more productive strategy than the sequential peptidic condensation of the two amino acids.

\section{Introduction}

Natural compounds with an indanone fragment, although relatively few in number, are very diverse both in functional groups present and in the biological activity. Among them (Figure 1), one can find toxic sesquiterpenes, named pterosins, contained in various fern species [1, 2] and usefull in diabet [3], osteoarthritis [4] and other diseases treatment; antibacterial polycondensed aromatic compounds such as indanonaftol B [5]; the biphenyl derivative 1 [6]; the dihydrofuranone derivative 2 [7]; as well as the carboxaldehyde derivative 3 with antitumor activity [8]. More generally, natural indanones or indanes can be considered as bio-inspiring or medicinal chemistry [9]. For example, indanone acetamides of type 4 were shown to exhibit promising antimicrobial and antifungal activity [10].

A remarkable indane drug, used in the treatment of neurodegenerative disorders, is Ladostigil (TV-3326), a reductive amination derivative of 6-hydroxyindan-1-one [11]. With the view to extending the scope of such bioactive compounds, methodological studies of the functionalization of the indanone core thus deserve particular attention.

Along this line, we previously reported on the functionalization of 6-hydroxyindan1-one with amino acid fragments through an oxyacetic linker, showing that the method of 
<smiles>Cc1cc2c(c(C)c1CCO)C(=O)C(C)(CO)C2</smiles><smiles>O=C1c2ccccc2C(O)C12Oc1ccc3cccc(O)c3c1O2</smiles>

Indanonaftol B

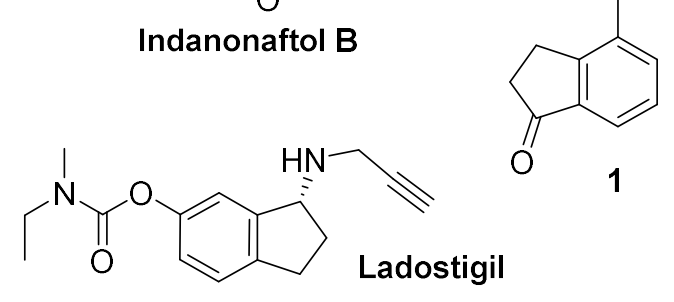<smiles>Cc1cc2c(c(C)c1CCCl)C(=O)C(C)C2</smiles><smiles>Cc1cc2c(c(C)c1CCCl)C(=O)C(C)C2</smiles>

Pterosin F

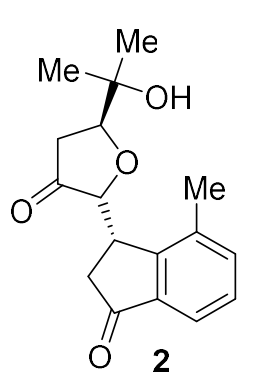<smiles>COC1CC(=O)c2c(C=O)cc(C)cc21</smiles><smiles>[R]N([R])C(=O)CC1CC(=O)c2ccccc21</smiles>

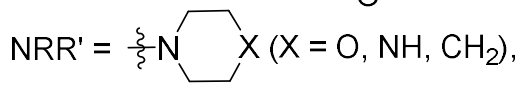<smiles>Cc1cc(=O)n(C(C)(C)n2cnc3ccccc32)n1-c1ccccc1</smiles>

Figure 1. Natural and synthetic indanone derivatives.

hydroxysuccinimide esters $[12,13]$ is more efficient than the carbonylimidazole condensation method [14].

The present report describes the beneficial use of hydroxysuccinimide esters for the synthesis of $\alpha$ - or $\beta$-dipeptide derivatives of 2-(3-oxo-2,3-dihydro-1H-inden-5-yloxy)acetic acid.

\section{Results and discussion}

Two possible strategies for the preparation of the $\alpha$ - or $\beta$-dipeptide targets can be distinguished: through either direct interaction of the indanone substrate with the terminal amino group of the preformed dipeptide, or sequential condensation of the two amino acids. The first method is obviously convenient but the second one can give more variety of products.

In order to explore the one-step strategy, the $N$-hydroxysuccinimide activated ester 6 was generated in situ from the acid 5 (Scheme 1), before addition of different dipeptides with a free amino function to obtain the target compounds 7a-i. TLC monitoring showed that the overall conversion was complete. Moderate to poor isolated yields were obtained in some cases (Scheme 1) because of the high solubility of the substance in the aqueous reaction medium (see Experimental part).

The two-step method was tested on tryptophan and methionine derivatives, the expected low solubility these substances $a$ priori can help to avoid the losses during workup and allowing a more accurate evaluation of the reaction efficiency. So, the carboxylic group of compounds 8a,b (Scheme 2, the synthesis was described in ref. [14]) was converted into the corresponding $\mathrm{N}$-hydroxysuccinimide activated ester 9. Without further purification, the latter was treated with another amino acid to give dipeptides $7 \mathbf{j}-\mathbf{n}$. The moderate overall yields of 


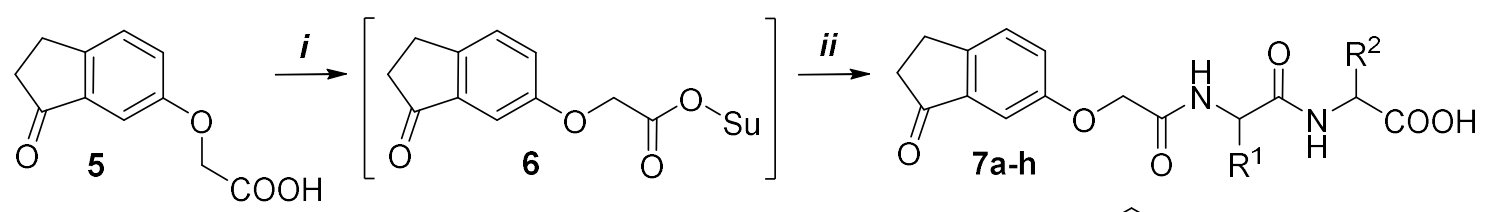

i: $1 . \longrightarrow(\mathrm{DCC})$, abs. dioxane, $\mathrm{rt} ; 2$.

ii: 1. dipeptide, $\mathrm{NaHCO}_{3}$, dioxane:water $1: 1, \mathrm{rt} ; 2 . \mathrm{HCl}$<smiles>CC(NC(=O)COc1ccc2c(c1)C(=O)CC2)C(=O)NCCC(=O)O</smiles>

7a Gly-Gly $\left(\mathrm{R}^{1}=\mathrm{H}, \mathrm{R}^{2}=\mathrm{H}\right) ; 7 \mathrm{~b}$ Gly-Ala $\left(\mathrm{R}^{1}=\mathrm{H}, \mathrm{R}^{2}=\mathrm{Me}\right) ; 7 \mathrm{c} \quad$ Gly-Val $\left(\mathrm{R}^{1}=\mathrm{H}, \mathrm{R}^{2}=i-\mathrm{Pr}\right)$;

7d Gly-Leu $\left(\mathrm{R}^{1}=\mathrm{H}, \mathrm{R}^{2}=i-\mathrm{Bu}\right) ; 7 \mathrm{e}$ Gly-Tyr $\left(\mathrm{R}^{1}=\mathrm{H}, \mathrm{R}^{2}=\mathrm{CH}_{2}\left(4-\mathrm{OHC}_{6} \mathrm{H}_{4}\right)\right) ; 7 \mathrm{f}$ Ala-Val $\left(\mathrm{R}^{1}=\mathrm{Me}, \mathrm{R}^{2}=i-\mathrm{Pr}\right)$;

$7 \mathrm{~g}$ Ala-Met $\left(\mathrm{R}^{1}=\mathrm{Me}, \mathrm{R}^{2}=\mathrm{CH}_{2} \mathrm{CH}_{2} \mathrm{SCH}_{3}\right) ; 7 \mathrm{~h}$ Ala-Trp $\left(\mathrm{R}^{1}=\mathrm{Me}, \mathrm{R}^{2}=\mathrm{CH}_{2}\right.$ (indol-3-yl)); $7 \mathrm{i}$ Ala- $\beta-$ Ala

Scheme 1. One-pot synthesis of the $\alpha$-dipeptide derivatives 7a-h and $\beta$-dipetide derivative $7 \mathbf{i}$ from the acid 5 and the corresponding pre-formed dipeptides.

products $7 \mathbf{j}-\mathbf{n}$ were due the low yields of the corresponding initial transformation $\mathbf{5} \rightarrow \mathbf{8}$ (see in ref. [14]), and the yield of the conversion $5 \rightarrow 7$ after two steps was only $26 \%$ for dipeptide $\mathbf{7 j}, 33 \%$ - for $\mathbf{7 k}, 25 \%$ - for $\mathbf{7 1}$, and $42 \%$ - for $7 \mathbf{m}, 34 \%$ - for $7 \mathbf{n}$ respectively.

All spectral data confirmed the structure of the products 7 (see Experimental part). The ${ }^{1} \mathrm{H}$ NMR spectra in DMSO- $d_{6}$ solutions did not allow detection of the $\mathrm{COOH}$ signals due to fast exchange; but the IR spectra displayed the corresponding wide intense absorption band of the $\mathrm{O}-\mathrm{H}$ bond, along with a maximum absorption of the $\mathrm{N}-\mathrm{H}$ bond near to $3300 \mathrm{~cm}^{-1}$.

The syntheses were performed from optically pure amino acids, except the synthesis of 71 for which optically pure L-tryptophan and racemic DL-alanine were used. So, substance 71 was obtained as a mixture of equal amounts of two diastereomers, as shown by ${ }^{1} \mathrm{H}$ NMR spectroscopy.

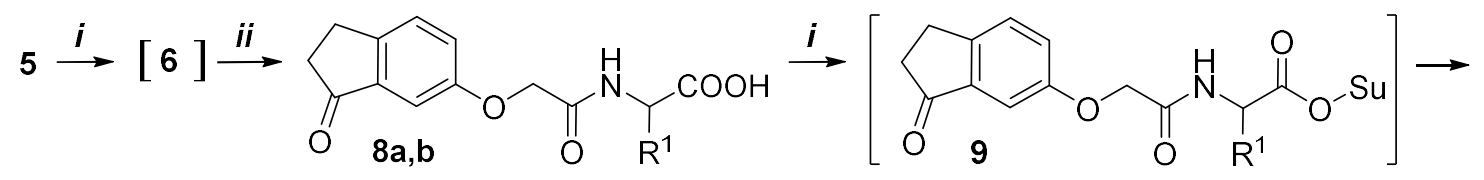<smiles>[R]C(NC(=O)C([R])NC(=O)COc1ccc2c(c1)C(=O)C(C#C)C2)C(=O)O</smiles>

i: 1. DCC, abs. dioxane, rt; 2 . Su-OH

ii: 1. amino acid, $\mathrm{NaHCO}_{3}$, dioxane:water $1: 1, \mathrm{rt} ; 2 . \mathrm{HCl}$<smiles>O=C(O)CCNC(=O)C(Cc1c[nH]c2ccccc12)NC(=O)COc1ccc2c(c1)C(=O)CC2</smiles>

7j Met-Trp $\left(\mathrm{CH}_{2} \mathrm{CH}_{2} \mathrm{SCH}_{3}, \mathrm{CH}_{2}\right.$ (indol-3-yl)); 7k Trp-Gly $\left(\mathrm{CH}_{2}\right.$ (indol-3-yl), $\left.\mathrm{H}\right) ;$ 7l Trp-Ala ( $\mathrm{CH}_{2}$ (indol-3-yl), Me); $7 \mathrm{~m} \operatorname{Trp}-\operatorname{Met}\left(\mathrm{CH}_{2}\right.$ (indol-3-yl), $\left.\mathrm{CH}_{2} \mathrm{CH}_{2} \mathrm{SCH}_{3}\right) ; 7$ Trp- $\beta$-Ala; 8a Met $\left(\mathrm{CH}_{2} \mathrm{CH}_{2} \mathrm{SCH}_{3}\right) ; 8$ b Trp $\left(\mathrm{CH}_{2}\right.$ (indol-3-yl))

Scheme 2. Two-step synthesis of the $\alpha$-dipeptide derivatives $\mathbf{7 j} \mathbf{j}-\mathbf{m}$ and $\beta$-dipetide derivative $\mathbf{7 n}$ from the acid $\mathbf{5}$ by consecutive reactions with two amino acids. 


\section{Conclusions}

It was shown that the dipeptide derivatives of 2-(3-oxo-2,3-dihydro- $1 H$-inden5-yloxy)acetic acid can be obtained by either consecutive reactions of the two amino acids, or immediately from a pre-formed dipeptide precursor. Nonetheless, the one-step strategy appears more efficient: both more convenient and more productive. A more exact quantitative estimate can be obtained subsequently using parallel synthesis of series of identical dipeptides by these two methods.

The developed synthetic procedures can be useful for obtaining a number of new indanone amino acid derivatives in order to study their optical characteristics and biological activity.

\section{Experimental part}

The reaction progress and identities of the products were controlled with TLC on Merck $F_{254}$ plates using a chloroform:methanol $(19: 1, v / v)$ eluting system.

${ }^{1} \mathrm{H}$ and ${ }^{13} \mathrm{C}$ NMR spectra were recorded on a Varian 400 spectrometer operating at $400 \mathrm{MHz}$ for ${ }^{1} \mathrm{H}$ and $100 \mathrm{MHz}$ for ${ }^{13} \mathrm{C}$. NMR chemical shifts are reported in ppm, in the $\delta$ scale and are referenced using TMS as internal standard.

FTIR spectra were recorded on a Thermo Nicolet Nexus 470 spectrometer in $\mathrm{KBr}$ pellets.
Melting points were determined using a Leica Galen III Kofler-type melting point microscope, and were uncorrected.

The synthesis of $\mathbf{5}$ was performed using a previously described procedure $[14,15]$; for the synthesis of the amino acid derivatives 8 , see ref. [14].

\section{General procedure for $\mathrm{N}$-acylation} through the $N$-hydroxysuccinimide ester method. To a solution of the acid $\mathbf{5 ,} \mathbf{8 a}$ or $\mathbf{8 b}$ $(1.5 \mathrm{mmol})$ and $N$-hydroxysuccinimide $(0.23 \mathrm{~g}$, $2 \mathrm{mmol})$ in absolute dioxane $(10 \mathrm{~mL})$ dicyclohexylcarbodiimide $(0.41 \mathrm{~g}, 2 \mathrm{mmol})$ was added at room temperature under vigorous stirring. The mixture was stirred at room temperature for 2-3 h until an activated ester was formed (TLC monitoring); then a solution of the corresponding amino acid or dipeptide $(1.75 \mathrm{mmol})$ and $\mathrm{NaHCO}_{3}(0.25 \mathrm{~g}, 3 \mathrm{mmol})$ in water $(10 \mathrm{~mL})$ was added (the amino acids and dipeptide were been used in free amine form and not in protonated form). The reaction mixture was stirred at room temperature for $2-3 \mathrm{~h}$ (TLC monitoring). After the process was finished, the dicyclohexylurea precipitate was filtered off. The filtrate was poured into water $(50 \mathrm{~mL})$ and the solution was acidified (to $\mathrm{pH} 4-5$ ) with dilute $\mathrm{HCl}$. The precipitate formed was filtered off, recrystallized from a $i$-PrOH:water 1:1 mixture.

$N$-\{[(1-oxoindan-6-yl)oxy $]$ acetyl $\}$ glycylglycine 7a. Yield $0.26 \mathrm{~g}, 0.81 \mathrm{mmol}, 54 \%$. Mp 229-230 ${ }^{\circ} \mathrm{C} .{ }^{1} \mathrm{H}$ NMR (DMSO- $\left.d_{6}\right), \quad \delta$ (ppm): 
2.63 (br. s, 2H, $\mathrm{H}_{2}-3$ ), 3.00 (br. s, $2 \mathrm{H}, \mathrm{H}_{2}-2$ ), 3.65-3.85 (br. s, $4 \mathrm{H}, 2 \mathrm{NHCH}_{2}$ ), 4.59 (s, $2 \mathrm{H}$, $\mathrm{OCH}_{2} \mathrm{CO}$ ), 7.10 (br. s, $1 \mathrm{H}, \mathrm{H}-7$ ), 7.33 (br. d, $J=6.8 \mathrm{~Hz}, 1 \mathrm{H}, \mathrm{H}-5), 7.50(\mathrm{~d}, J=6.8 \mathrm{~Hz}, 1 \mathrm{H}, \mathrm{H}-$ 4), 8.23 (br. s, $1 \mathrm{H}, \mathrm{NH}$ ), 8.39 (br. s, 1H, NH). ${ }^{13} \mathrm{C}$ NMR (DMSO- $\left.d_{6}\right), \quad \delta$ (ppm): 25.1, 37.1, 41.1, 42.0, 67.6, 106.7, 123.8, 128.3, 138.2, $148.8,157.7,168.2,169.4,171.5,206.2$. IR $(\mathrm{KBr}), v\left(\mathrm{~cm}^{-1}\right): 3289,3099,2934,1714,1671$, $1633,1569,1492,1438,1288,1253,1070,834$, $746,558$.

$N$-\{[(1-oxoindan-6-yl)oxy $]$ acetyl $\}$ glycylalanine $7 \mathbf{b}$. Yield $0.24 \mathrm{~g}, 0.72 \mathrm{mmol}, 48 \%$. Mp 202-203 ${ }^{\circ} \mathrm{C}$. ${ }^{1} \mathrm{H}$ NMR (DMSO- $d_{6}$ ), $\delta$ (ppm): 1.25 (d, $J=7.0 \mathrm{~Hz}, 3 \mathrm{H}, \mathrm{CHCH}_{3}$ ), 2.63 (br. s, $2 \mathrm{H}$, $\mathrm{H}_{2}-3$ ), 3.00 (br. s, $2 \mathrm{H}, \mathrm{H}_{2}-2$ ), 3.72-3.85 (br. s, $\left.4 \mathrm{H}, \mathrm{NHCH}_{2}\right), 4.16-4.25\left(\mathrm{~m}, 1 \mathrm{H}, \underline{\mathrm{CHCH}_{3}}\right), 4.59$ (s, 2H, $\mathrm{OCH}_{2} \mathrm{CO}$ ), 7.09 (br. s, 1H, H-7), 7.32 (br. d, $J=8.0 \mathrm{~Hz}, 1 \mathrm{H}, \mathrm{H}-5), 7.50$ (d, $J=8.0 \mathrm{~Hz}$, $1 \mathrm{H}, \mathrm{H}-4), 8.24$ (d, J=7.0 Hz, 1H, $\underline{\mathrm{NHCH}}$ ), 8.32

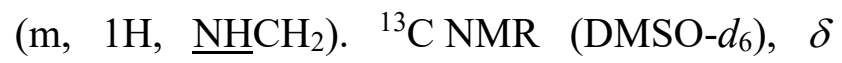
(ppm): 17.6, 25.1, 37.0, 41.8, 48.0, 67.5, 106.8, 123.8, 128.2, 138.2, 148.8, 157.7, 168.1, 168.6, 174.4, 206.4. IR (KBr), $v\left(\mathrm{~cm}^{-1}\right): 3284,3089$, 2928, 1706, 1665, 1648, 1561, 1491, 1438, $1285,1224,1065,835,720,559$.

$N$-\{[(1-oxoindan-6-yl)oxy]acetyl $\}$ glycylvaline 7c. Yield $0.25 \mathrm{~g}, 0.69 \mathrm{mmol}, 46 \%$. Mp $159-159^{\circ} \mathrm{C} . \quad{ }^{1} \mathrm{H}$ NMR (DMSO- $\left.d_{6}\right), \delta$ (ppm): 0.85 (br. s, $\left.6 \mathrm{H}, \mathrm{CHCH}\left(\mathrm{CH}_{3}\right)_{2}\right), 1.98-2.09$ (m, $\left.1 \mathrm{H}, \mathrm{CHCH}\left(\mathrm{CH}_{3}\right)_{2}\right), 2.62$ (br. s, 2H, $\mathrm{H}_{2}-3$ ), 2.99 (br. s, $2 \mathrm{H}, \mathrm{H}_{2}-2$ ), 3.80-3.91 (br. s, $4 \mathrm{H}, \mathrm{NHCH}_{2}$ ), 4.11-4.20 (m, 1H, $\left.\underline{\mathrm{CHCH}}\left(\mathrm{CH}_{3}\right)_{2}\right), 4.58(\mathrm{~s}, 2 \mathrm{H}$,
$\mathrm{OCH}_{2} \mathrm{CO}$ ), 7.09 (br. s, 1H, H-7), 7.32 (br. d, $J=6.0 \mathrm{~Hz}, 1 \mathrm{H}, \mathrm{H}-5), 7.50(\mathrm{~d}, J=6.0 \mathrm{~Hz}, 1 \mathrm{H}, \mathrm{H}-$ 4), 8.04 (d, J=6.8 Hz, 1H, $\mathrm{NHCH}), 8.32$ (m, $1 \mathrm{H}$,

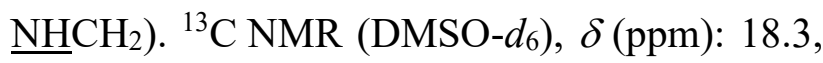
19.5, 25.1, 30.4, 37.0, 41.9, 57.7, 67.5, 106.6, $123.8,128.2,138.2,148.8,157.7,168.1,169.0$, 173.4, 206.2. IR (KBr), $v\left(\mathrm{~cm}^{-1}\right): 3293,3057$, 2965, 2929, 1707, 1663, 1544, 1490, 1284, 1224, 1067, 837, 559.

$N$ - $\{[(1-$ oxoindan-6-yl)oxy]acetyl $\}$ glycylleucine 7d. Yield 0,45 g, $1.2 \mathrm{mmol}, 80 \%$. Mp $131-132^{\circ} \mathrm{C} .{ }^{1} \mathrm{H}$ NMR (DMSO- $d_{6}$ ), $\delta$ (ppm): 0.83 (d, $\left.J=6.0 \mathrm{~Hz}, 3 \mathrm{H}, \mathrm{CH}_{3}\right), 0.87$ (d, $J=6.0 \mathrm{~Hz}, 3 \mathrm{H}$, $\left.\mathrm{CH}_{3}\right), 1.51$ (t, J=6.6 Hz, $\left.2 \mathrm{H}, \mathrm{CHCH} 2 \mathrm{CH}\left(\mathrm{CH}_{3}\right)_{2}\right)$, 1.57-1.67 (m, $\left.1 \mathrm{H}, \mathrm{CHCH}_{2} \underline{\mathrm{CH}}\left(\mathrm{CH}_{3}\right)_{2}\right), 2.63$ (br. t, $J=4.8 \mathrm{~Hz}, 2 \mathrm{H}, \mathrm{H}_{2}-3$ ), 3.01 (br. t, $J=4.8 \mathrm{~Hz}$, $2 \mathrm{H}, \mathrm{H}_{2}-2$ ), 3.81 (br. d, J=5.0 Hz, $\mathrm{NHCH}_{2}$ ), 4.25

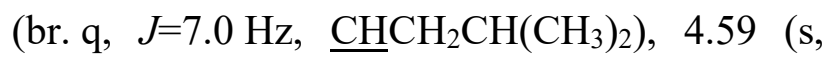
$2 \mathrm{H}, \mathrm{OCH}_{2} \mathrm{CO}$ ), 7.11 (br. s, $1 \mathrm{H}, \mathrm{H}-7$ ), 7.32 (br. d, $J=8.2 \mathrm{~Hz}, 1 \mathrm{H}, \mathrm{H}-5), 7.50(\mathrm{~d}, J=8.2 \mathrm{~Hz}, 1 \mathrm{H}, \mathrm{H}-$ 4), 8.24 (d, $J=7.0 \mathrm{~Hz}, 1 \mathrm{H}, \underline{\mathrm{NHCH}}), 8.32$ (br. t, $J=5.0 \mathrm{~Hz}, 1 \mathrm{H}, \underline{\mathrm{NHCH}} 2) .{ }^{13} \mathrm{C}$ NMR (DMSO- $d_{6}$ ), $\delta(\mathrm{ppm}): 21.8,23.2,24.7,25.1,37.0,41.8,50.7$, $67.6,106.8,123.8,128.2,138.2,148.8,157.7$, 168.1, 168.8, 174.4, 206.4. IR ( $\mathrm{KBr}), v\left(\mathrm{~cm}^{-1}\right)$ : 3303, 2958, 2928, 1707, 1664, 1545, 1490, 1284, 1224, 1067, 837.

$N$ - $\{[(1-$ oxoindan-6-yl)oxy $]$ acetyl $\}$ glycyltyrosine 7e. Yield $0.51 \mathrm{~g}, 1.2 \mathrm{mmol}, 80 \%$. Mp $138-139^{\circ} \mathrm{C} .{ }^{1} \mathrm{H}$ NMR (DMSO- $\left.d_{6}\right), \quad \delta$ (ppm): 2.64 (br. t, J=5.2 Hz, 2H, $\mathrm{H}_{2}-3$ ), 2.76 (dd, $\left.J=13.6 \mathrm{~Hz}, J=9.0 \mathrm{~Hz}, 1 \mathrm{H}, \underline{\mathrm{CH}}_{\alpha}-\mathrm{CH}(\mathrm{COOH})\right)$, $2.92\left(\mathrm{dd}, J=13.6 \mathrm{~Hz}, J=4.7 \mathrm{~Hz}, 1 \mathrm{H}, \underline{\mathrm{CH}_{\beta}}-\right.$ 
$\mathrm{CH}(\mathrm{COOH})$ ), 3.01 (br. $\mathrm{t}, J=5.2 \mathrm{~Hz}, 2 \mathrm{H}, \mathrm{H}_{2}-3$ ), $3.71\left(\mathrm{dd}, J=16.5 \mathrm{~Hz}, J=5.7 \mathrm{~Hz}, 1 \mathrm{H}, \mathrm{NH}-\underline{\mathrm{CH}}_{\alpha}-\right.$ CONH), 3.81 (dd, $J=16.5 \mathrm{~Hz}, J=5.7 \mathrm{~Hz}, 1 \mathrm{H}$, $\left.\mathrm{NH}-\underline{\mathrm{CH}} \beta{ }^{-} \mathrm{CONH}\right), \quad 4.30-4.37\left(\mathrm{~m}, 1 \mathrm{H}, \mathrm{CH}_{2}-\right.$ $\underline{\mathrm{CH}}(\mathrm{COOH})), 4.58\left(\mathrm{~s}, 2 \mathrm{H}, \mathrm{OCH}_{2} \mathrm{CO}\right), 6.64$ (d, $\left.J=8.2 \mathrm{~Hz}, 2 \mathrm{H}, \mathrm{H}-3^{\prime}, 5^{\prime}\left(4-\mathrm{HOC}_{6} \mathrm{H}_{4}\right)\right), 6.99$ (d, $\left.J=8.2 \mathrm{~Hz}, 2 \mathrm{H}, \mathrm{H}-2^{\prime}, 6^{\prime}\left(4-\mathrm{HOC}_{6} \mathrm{H}_{4}\right)\right), 7.11$ (br. s, 1H, H-7), 7.33 (br. d, J=8.6 Hz, H-5), 7.50 (d, $J=8.6 \mathrm{~Hz}, \mathrm{H}-4), 8.15$ (d, $J=7.8 \mathrm{~Hz}, 1 \mathrm{H}, \underline{\mathrm{NHCH}})$, $8.31\left(\mathrm{t}, J=5.7 \mathrm{~Hz}, \quad 1 \mathrm{H}, \quad \underline{\left.\mathrm{NHCH}_{2}\right) .}{ }^{13} \mathrm{C} \mathrm{NMR}\right.$ (DMSO- $\left.d_{6}\right), \delta$ (ppm): 25.2, 36.6, 37.1, 41.9, 54.4, 67.5, 106.8, 115.4, 123.9, 127.9, 130.5, 138.2, 148.8, 156.3, 157.7, 168.1, 168.7, 173.4, 186.9, 206.4. IR (KBr), v (cm $\left.{ }^{-1}\right): 3291,2925$, 1706, 1662, 1547, 1516, 1490, 1438, 1284, 1247, 1064, 835, 558.

$N$ - $\{[(1-o x o i n d a n-6-y l) o x y]$ acetyl $\}$ alanylvaline 7f. Yield $0.28 \mathrm{~g}, 0.75 \mathrm{mmol}, 50 \%$. Mp 174- $175^{\circ} \mathrm{C} .{ }^{1} \mathrm{H}$ NMR (DMSO- $\left.d_{6}\right), \delta(\mathrm{ppm})$ : 0.81-0.87 (m, 6H, $\left.\mathrm{CHCH}\left(\underline{\mathrm{CH}}_{3}\right)_{2}\right), 1.99-2.10$ $\left(\mathrm{m}, 1 \mathrm{H}, \mathrm{CH} \underline{\mathrm{CH}}\left(\mathrm{CH}_{3}\right)_{2}\right), 1.25(\mathrm{~d}, J=6.6 \mathrm{~Hz}, 3 \mathrm{H}$, $\mathrm{CHCH}_{3}$ ), 2.63 (m, 2H, H2-3), 3.00 (m, 2H, H22), 4.18 (br. t, $\left.J=6.5 \mathrm{~Hz}, 1 \mathrm{H}, \underline{\mathrm{CHCH}}\left(\mathrm{CH}_{3}\right)_{2}\right)$, 4.59 (m, 3H, $\left.\mathrm{CHCH}_{3}, \mathrm{OCH}_{2} \mathrm{CO}\right), 7.08$ (br. s, 1H, H-7), 7.30 (br. d, J=8.2 Hz, H-5), 7.49 (d, $J=8.2 \mathrm{~Hz}, \mathrm{H}-4), 8.14$ (d, J=7.0 Hz, 1H, NH), 8.19 (d, $J=7.0 \mathrm{~Hz}, 1 \mathrm{H}, \mathrm{NH}) .{ }^{13} \mathrm{C}$ NMR (DMSO$\left.d_{6}\right), \delta$ (ppm): 18.0, 19.5, 25.1, 30.5, 37.0, 48.2, 57.3, 67.3, 106.3, 123.8, 128.2, 138.2, 148.7, 157.7, 167.2, 172.6, 173.3, 206.4. IR (KBr), v $\left(\mathrm{cm}^{-1}\right): 3277,3068,2967,2934,1740,1701$, 1654, 1549, 1491, 1443, 1267, 1227, 1077, 834, 676,561 .
$N$ - $\{[(1-o x o i n d a n-6-y l) o x y]$ acetyl $\}$ alanylmethionine 7g. Yield $0.20 \mathrm{~g}, 0.50 \mathrm{mmol}, 33 \%$. Mp $82-83^{\circ} \mathrm{C} .{ }^{1} \mathrm{H}$ NMR (DMSO- $\left.d_{6}\right), \delta$ (ppm): 1.25 (br. s, 3H, $\left.\mathrm{CHCH}_{3}\right), 1.79-2.06$ (m, 5H, $\left.\mathrm{CH}_{2} \underline{\mathrm{CH}}_{2} \underline{\mathrm{SCH}}_{3}\right), 2.44\left(\mathrm{~m}, 2 \mathrm{H}, \underline{\mathrm{CH}_{2}} \mathrm{CH}_{2} \mathrm{SCH}_{3}\right)$, 2.63 (br. s, 2H, $\mathrm{H}_{2}-3$ ), 3.00 (br. s, 2H, $\mathrm{H}_{2}-2$ ), $4.32(\mathrm{~m}, 1 \mathrm{H}, \mathrm{NHCHCO}), 4.41(\mathrm{~m}, 1 \mathrm{H}$, $\mathrm{NH} \underline{\mathrm{CHCO}}, 4.59$ (s, 2H, $\left.\mathrm{OCH}_{2} \mathrm{CO}\right), 7.10$ (br. s, 1H, H-7), 7.33 (br. d, J=6.0 Hz, 1H, H-5), 7.50 (d, $J=6.0 \mathrm{~Hz}, 1 \mathrm{H}, \mathrm{H}-4), 8.13$ (br. s, 1H, NH), 8.27 (br. s, $1 \mathrm{H}, \mathrm{NH}) .{ }^{13} \mathrm{C}$ NMR (DMSO- $\left.d_{6}\right), \delta$ (ppm): 15.0, 25.1, 30.1, 31.2, 37.0, 41.2, 48.4, 51.3, 67.4, 106.6, 123.8, 128.2, 138.2, 148.7, 157.8, 167.3, 172.4, 173.6, 206.4. IR (KBr), v $\left(\mathrm{cm}^{-1}\right): 3311,3064,2922,1724,1690,1541$, 1490, 1438, 1284, 1251, 1178, 1068, 837, 559.

$N$ - $\{[(1-o x o i n d a n-6-y l) o x y]$ acetyl $\}$ alanyltryptophane $7 \mathbf{h}$. Yield $0.35 \mathrm{~g}, 0.76 \mathrm{mmol}, 51 \%$. Mp $118-119^{\circ} \mathrm{C} .{ }^{1} \mathrm{H}$ NMR (DMSO- $\left.d_{6}\right), \delta(\mathrm{ppm})$ : $1.08\left(\mathrm{~d}, J=6.0 \mathrm{~Hz}, 1.5 \mathrm{H}, \mathrm{CHCH}_{3}\right), 1.23$ (d, $J=6.0 \mathrm{~Hz}, 1.5 \mathrm{H}, \mathrm{CHCH}_{3}$ ), 2.62 (br. s, $2 \mathrm{H}, \mathrm{H}_{2}-3$ ), 2.95-3.09 (m, 3H, $\mathrm{H}_{2}-2, \underline{\mathrm{CH}_{\alpha}}($ indol-3-yl)), 3.14-3.23 (m, 1H, $\left.\underline{\mathrm{CH}}_{\beta}(\mathrm{indol}-3-\mathrm{yl})\right), 4.32-4.58$ (m, 2H, 2 NHCHCO), 4.57 (m, 2H, $\left.\mathrm{OCH}_{2} \mathrm{CO}\right)$, 6.97 (br. t, $J=6.6 \mathrm{~Hz}, 1 \mathrm{H}, \mathrm{H}-5^{\prime}$ (indol-3-yl)), 7.01-7.09 (m, 2H, H-7, H-6' (indol-3-yl)), 7.14 (d, $J=13.5 \mathrm{~Hz}, 1 \mathrm{H}, \mathrm{H}-5), 7.27-7.34$ (m, 2H, H4, H-2' (indol-3-yl)), 7.47 (d, J=8.0 Hz, 1H, H4' (indol-3-yl)), 7.50-7.55 (m, 1H, H-7' (indol3-yl)), 8.10-8.19 (m, 1H, NH), 8.26 and 8.30 (two d, $J=6.7 \mathrm{~Hz}, 1 \mathrm{H}, \mathrm{NH}$ ), 10.87 (br. s, 1H, NH (indol-3-yl)). ${ }^{13} \mathrm{C}$ NMR (DMSO- $\left.d_{6}\right), \delta$ (ppm): 18.8, 25.2, 27.4, 37.1, 48.1, 53.5, 67.4, 106.7, 
$110.2,111.8,118.6,118.8,121.3,123.9,124.1$, $127.7,128.3,136.5,138.2,147.3,148.8,157.8$, 167.3, 173.6, 206.4. IR (KBr), $v\left(\mathrm{~cm}^{-1}\right): 3337$, 3058, 2927, 1686, 1665, 1560, 1490, 1277, 1220, 1099, 745, 559.

$N-\{[(1-o x o i n d a n-6-y l) o x y]$ acetyl $\}$ alanyl$\beta$-alanine 7i. Yield $0.27 \mathrm{~g}, 0.78 \mathrm{mmol}, 52 \%$. Mp $117-118^{\circ} \mathrm{C} .{ }^{1} \mathrm{H}$ NMR (DMSO- $\left.d_{6}\right), \delta(\mathrm{ppm})$ : 1.20 (d, J=6.0 Hz, 3H, $\mathrm{CHCH}_{3}$ ), 2.35 (br. t, $J=6.0 \mathrm{~Hz}, 2 \mathrm{H}, \mathrm{NHCH}_{2} \underline{\mathrm{CH}}_{2}$ ), 2.62 (br. s, $2 \mathrm{H}, \mathrm{H}_{2}-$ 3), 2.99 (br. s, $2 \mathrm{H}, \mathrm{H}_{2}-2$ ), 3.23 (br. t, $J=6.0 \mathrm{~Hz}$, $2 \mathrm{H}, \mathrm{NHCH}_{2} \mathrm{CH}_{2}$ ), 4.29 (br. t, $J=6.6 \mathrm{~Hz}, 1 \mathrm{H}$, $\left.\underline{\mathrm{CHCH}}\left(\mathrm{CH}_{3}\right)_{2}\right), 4.57\left(\mathrm{~s}, 2 \mathrm{H}, \mathrm{OCH}_{2} \mathrm{CO}\right), 7.06$ (br. s, 1H, H-7), 7.30 (br. d, J=8.0 Hz, 1H, H-5), 7.48 (d, $J=8.0 \mathrm{~Hz}, 1 \mathrm{H}, \mathrm{H}-4), 8.13$ (br. s, $1 \mathrm{H}$,

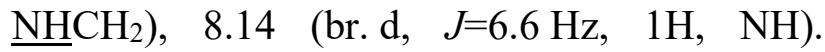
${ }^{13} \mathrm{C}$ NMR (DMSO- $\left.d_{6}\right), \quad \delta(\mathrm{ppm}): 18.9,25.1$, $34.2,35.3,37.0,48.3,67.3,106.3,123.9,128.2$, 138.2, 148.7, 157.7, 167.3, 172.3, 173.3, 206.5. IR $(\mathrm{KBr}), v\left(\mathrm{~cm}^{-1}\right): 3291,2929,1719,1693$, 1664, 1627, 1546, 1492, 1286, 1227, 1075, 833.

$N$ - $\{[(1-o x o i n d a n-6-y l) o x y]$ acetyl $\}$ -

methionyltryptophan $\mathbf{7 j}$. Yield $0.26 \mathrm{~g}$, $0.50 \mathrm{mmol}, 33 \%$. Mp $109-110^{\circ} \mathrm{C} .{ }^{1} \mathrm{H}$ NMR (DMSO- $\left.d_{6}\right), \quad \delta$ (ppm): 1.67-2.10 (m, 5H, $\mathrm{CH}_{2} \underline{\mathrm{CH}}_{2} \underline{\mathrm{SCH}}_{3}$ ), 2.62 (br. t, J=5.4 Hz, 2H, $\mathrm{H}_{2}-3$ ), $2.47\left(\mathrm{~m}, 2 \mathrm{H}, \underline{\mathrm{CH}_{2}} \mathrm{CH}_{2} \mathrm{SCH}_{3}\right), 2.98-3.13(\mathrm{~m}, 3 \mathrm{H}$, $\mathrm{H}_{2}-2, \underline{\mathrm{CH}_{\alpha}}($ indol-3-yl $), 3.18-3.25(\mathrm{~m}, 1 \mathrm{H}$, $\underline{\mathrm{CH}}_{\beta}\left(\right.$ indol-3-yl)), 4.43-4.61 (m, 4H, $\mathrm{OCH}_{2} \mathrm{CO}$, $2 \mathrm{NHCHCO}$ ), 6.94 (br. t, $J=7.4 \mathrm{~Hz}, 1 \mathrm{H}, \mathrm{H}-5^{\prime}$ (indol-3-yl)), 7.01 (br. t, J=7.4 Hz, 1H, H-6' (indol-3-yl)), 7.05-7.13 (m, 2H, H-5,7), 7.247.32 (m, 2H, H-4, H-2' (indol-3-yl)), 7.42 (d,
$J=8.5 \mathrm{~Hz}, 1 \mathrm{H}, \mathrm{H}-4^{\prime}$ (indol-3-yl)), 7.49-7.54 (m, $1 \mathrm{H}, \mathrm{H}-7^{\prime}$ (indol-3-yl)), 8.05-8.21 and 8.32-8.41 (two m, 1.5 and $0.5 \mathrm{H}, 2 \mathrm{NH}$ ), 10.74 (br. s, 1H, $\mathrm{NH}$ (indol-3-yl)). ${ }^{13} \mathrm{C}$ NMR $\quad\left(\right.$ DMSO- $\left.d_{6}\right), \quad \delta$ (ppm): 15.0, 25.1, 29.7, 32.5, 36.9, 51.8, 53.4, $62.4,67.2,106.5,110.0,111.8,118.5,118.8$, $121.3,123.7,124.0,127.6,128.3,136.5,138.2$, $148.7,157.7,167.7,171.0,173.6,206.4$. IR $(\mathrm{KBr}), v\left(\mathrm{~cm}^{-1}\right): 3324,3057,2922,1701,1665$, 1533, 1489, 1442, 1277, 1220, 1061, 837, 745, 560.

\section{$N$-\{[(1-oxoindan-6-yl)oxy $]$ acetyl $\}$ -}

tryptophanylglycine $7 \mathbf{k}$. Yield $\quad 0.39 \mathrm{~g}$, $0.87 \mathrm{mmol}, 58 \%$. $\mathrm{Mp} 139-140^{\circ} \mathrm{C} .{ }^{1} \mathrm{H}$ NMR (DMSO-d $\left.d_{6}\right), \delta$ (ppm): 2.63 (br. s, 2H, $\mathrm{H}_{2}-3$ ), 3.01-3.25 (m, 4H, H2-2, $\underline{\mathrm{CH}}_{2}$ (indol-3-yl)), 3.73 (br. s, 2H, $\left.\mathrm{NHCH}_{2}\right), \quad 4.40-4.52$ (m, 2H, $\left.\mathrm{OCH}_{2} \mathrm{CO}\right), 4.62-4.71$ (m, 1H, NHㄷC), 6.93 (br. t, $J=6.0 \mathrm{~Hz}, 1 \mathrm{H}, \mathrm{H}-5^{\prime}$ (indol-3-yl)), 6.997.05 (m, 2H, H-7, H-6' (indol-3-yl)), 7.08-7.14 (m, 2H, H-5, H-2' (indol-3-yl)), 7.30 (d, $J=7.0 \mathrm{~Hz}, 1 \mathrm{H}, \mathrm{H}-4), 7.38$ (d, $J=7.6 \mathrm{~Hz}, 1 \mathrm{H}, \mathrm{H}-4^{\prime}$ (indol-3-yl)), 7.57 (d, J=7.6 Hz, 1H, H-7' (indol-3-yl)), 7.94 (d, $J=7.4 \mathrm{~Hz}, 1 \mathrm{H}, \mathrm{NH}), 8.31-$ 8.35 (m, 1H, NH), 10.64 (br. s, 1H, NH (indol3-yl)). $\left.{ }^{13} \mathrm{C} \mathrm{NMR} \mathrm{(DMSO-} d_{6}\right), \delta$ (ppm): 25.1, 28.0, 37.0, 41.2, 53.5, 67.5, 106.6, 110.2, 111.8, $118.6 \times 2,121.2,123.5,124.2,127.8,128.2$, 136.4, 138.1, 148.7, 157.6, 167.6, 171.6, 171.9, 206.2. IR (KBr), $v\left(\mathrm{~cm}^{-1}\right): 3313,3057,2927$, 1711, 1664, 1541, 1489, 1437, 1278, 1221, $1063,835,795,743,559$. 
$N$-\{[(1-oxoindan-6-yl)oxy]acetyl $\}$ -

tryptophanylalanine 7l. Yield $\quad 0.30 \mathrm{~g}$, $0.65 \mathrm{mmol}, 43 \%$. Mp $139-141^{\circ} \mathrm{C}$. ${ }^{1} \mathrm{H}$ NMR (DMSO- $d_{6}$ ), $\delta$ (ppm): 1.18 (br. s, $1.5 \mathrm{H}, \mathrm{CHCH}_{3}$ ), 1.29 (br. s, $1.5 \mathrm{H}, \mathrm{CHCH}_{3}$ ), 2.63 (br. s, $2 \mathrm{H}, \mathrm{H}_{2}-$ 3), 2.96-3.29 (m, $2 \mathrm{H}, \mathrm{H}_{2}-2, \underline{\mathrm{CH}}_{2}$ (indol-3-yl)), 4.10-4.29 (m, 1H, NHCHCO), 4.42-4.72 (m, $\left.3 \mathrm{H}, \mathrm{OCH}_{2} \mathrm{CO}, \mathrm{NHCHCO}\right), 6.91-7.23(\mathrm{~m}, 5 \mathrm{H}$, $\mathrm{H}-5,7, \mathrm{H}-2^{\prime}, 5^{\prime}, 6^{\prime}$ (indol-3-yl)), 7.30-7.37 (m, 2H, H-4), 7.42-7.49 (m, 1H, H-4' (indol-3-yl)), 7.56-7.66 (m, 1H, H-7' (indol-3-yl)), 8.00-8.19 (m, 1H, NH), 8.30-8.47 (m, 1H, NH), 10.82 (br. s, 1H, NH (indol-3-yl)). ${ }^{13} \mathrm{C}$ NMR (DMSO$\left.d_{6}\right), \delta(\mathrm{ppm}): 17.2,25.2,28.1,37.0,48.2,53.3$, 67.4, 106.7, 109.9, 111.6, 118.6, 118.8, 121.2, $123.5,124.2,127.8,128.2,136.5,138.2,148.5$, 157.4, 167.4, 171.2, 174.5, 206.4. IR (KBr), v $\left(\mathrm{cm}^{-1}\right): 3316,3057,2927,1668,1532,1489$, 1445, 1277, 1221, 1061, 836, 745, 559.

$N$-\{[(1-oxoindan-6-yl)oxy]acetyl $\}$ -

tryptophanylmethionine $7 \mathbf{m}$. Yield $\quad 0.57 \mathrm{~g}$, $1.1 \mathrm{mmol}, \quad 73 \%$. Mp $111-112^{\circ} \mathrm{C} .{ }^{1} \mathrm{H}$ NMR (DMSO- $\left.d_{6}\right), \quad \delta$ (ppm): 1.83-2.05 (m, 5H, $\left.\mathrm{CH}_{2} \mathrm{CH}_{2} \mathrm{SCH}_{3}\right), 2.17-2.32$ (m, $1 \mathrm{H}, \underline{\mathrm{CH}}_{\alpha} \mathrm{CH}_{2} \mathrm{SCH}_{3}$ ),

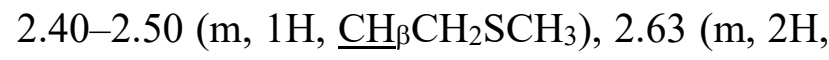
$\left.\mathrm{H}_{2}-3\right), 2.96-3.08$ ( $\mathrm{m}, 3 \mathrm{H}, \mathrm{H}_{2}-2, \underline{\mathrm{CH}} \alpha($ indol-3yl)), 3.09-3.22 (m, 1H, $\underline{\mathrm{CH}}_{\beta}($ indol-3-yl)), 4.23$4.30\left(\mathrm{~m}, 0.5 \mathrm{H}, \underline{\mathrm{CH}}\left(\mathrm{CH}_{2}\right)_{2} \mathrm{SCH}_{3}\right), 4.32-4.39(\mathrm{~m}$, $\left.0.5 \mathrm{H}, \underline{\mathrm{CH}}\left(\mathrm{CH}_{2}\right)_{2} \mathrm{SCH}_{3}\right), \quad 4.44-4.59(\mathrm{~m}, 2 \mathrm{H}$, $\mathrm{OCH}_{2} \mathrm{CO}$ ), 4.59-4.73 (m, 1H, $\underline{\mathrm{CHCH}}_{2}$ (indol-3yl), 6.95 (br. t, $J=7.6 \mathrm{~Hz}, 1 \mathrm{H}, \mathrm{H}-5^{\prime}$ (indol-3-yl)), 7.01-7.08 (m, 2H, H-7, H-6' (indol-3-yl)), 7.127.22 (m, 2H, H-5, H-2' (indol-3-yl)), 7.31 (d,
$J=8.0 \mathrm{~Hz}, 1 \mathrm{H}, \mathrm{H}-4), 7.44$ (d, $J=8.0 \mathrm{~Hz}, 1 \mathrm{H}, \mathrm{H}-4^{\prime}$ (indol-3-yl)), 7.62 (d, J=8.0 Hz, 1H, H-7' (indol-3-yl)), $8.09(\mathrm{~d}, J=8.0 \mathrm{~Hz}, 0.5 \mathrm{H}, \mathrm{NH}$ ), $8.13(\mathrm{~d}, J=8.0 \mathrm{~Hz}, 0.5 \mathrm{H}, \mathrm{NH}), 8.42-8.48(\mathrm{~m}$, $1 \mathrm{H}, \mathrm{NH}), 10.80-10.87(\mathrm{~m}, 1 \mathrm{H}, \mathrm{NH}$ (indol-3yl)). ${ }^{13} \mathrm{C}$ NMR (DMSO- $d_{6}$ ), $\delta$ (ppm): 14.9, 25.1, 30.1, 31.2, 37.0, 51.6, 53.6, 66.8, 67.5, 106.9, $110.1,111.8,118.8,118.9,121.4,123.7,124.5$, $127.9,128.4,136.5,138.2,148.7,157.7,167.5$, 171.7, 173.6, 206.3. IR (KBr), v $\left(\mathrm{cm}^{-1}\right): 3320$, 3057, 2922, 2853, 1663, 1534, 1489, 1438, 1277, 1221, 1062, 871, 744, 559.

\section{$N$-\{[(1-oxoindan-6-yl)oxy]acetyl $\}$ -}

tryptophanyl- $\beta$-alanine $7 \mathbf{n}$. Yield $\quad 0.44 \mathrm{~g}$, $0.95 \mathrm{mmol}, 63 \%$. Mp $126-127^{\circ} \mathrm{C} .{ }^{1} \mathrm{H}$ NMR (DMSO- $\left.d_{6}\right), \delta$ (ppm): 2.32 (t, $J=7.0 \mathrm{~Hz}, 2 \mathrm{H}$, $\mathrm{NHCH}_{2} \underline{\mathrm{CH}}_{2}$ ), 2.64 (br. t, $J=5.6 \mathrm{~Hz}, 2 \mathrm{H}, \mathrm{H}_{2}-3$ ), 2.95-3.06 (3H, m, $\mathrm{H}_{2}-2$, $\underline{\mathrm{CH}}_{\alpha}($ indol-3-yl)), 3.12

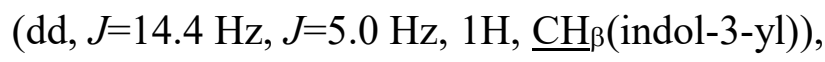
3.19-3.28 (m, $\left.2 \mathrm{H}, \mathrm{NHCH}_{2} \mathrm{CH}_{2}\right), 4.45-4.57$ (m,

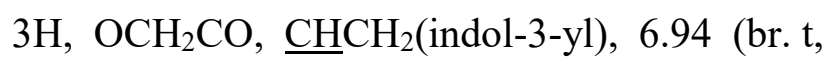
$J=7.6 \mathrm{~Hz}, 1 \mathrm{H}, \mathrm{H}-5^{\prime}$ (indol-3-yl)), 7.02-7.07 (m, 2H, H-7, H-6' (indol-3-yl)), 7.11 (br. s, 1H, H-2' (indol-3-yl)), 7.19 (dd, $J=8.0 \mathrm{~Hz}, J=2.4 \mathrm{~Hz}, 1 \mathrm{H}$, $\mathrm{H}-5), 7.31$ (d, J=8.0 Hz, 1H, H-4), 7.45 (d, $J=7.6 \mathrm{~Hz}, \quad 1 \mathrm{H}, \quad \mathrm{H}-4^{\prime}$ (indol-3-yl)), 7.58 (d, $J=7.6 \mathrm{~Hz}, \quad 1 \mathrm{H}, \quad \mathrm{H}-7^{\prime} \quad$ (indol-3-yl)), 8.09 (d, $J=8.0 \mathrm{~Hz}, 1 \mathrm{H}, \mathrm{NH}$ ), 8.17 (br. t $J=5.0 \mathrm{~Hz}, 1 \mathrm{H}$, $\mathrm{NH}), 10.81$ (s, 1H, NH (indol-3-yl)). ${ }^{13} \mathrm{C}$ NMR (DMSO- $\left.d_{6}\right), \delta$ (ppm): 25.1, 28.3, 34.2, 35.4, 37.0, 53.6, 67.3, 106.7, 110.1, 111.6, 118.7, $118.9,121.4,123.8,124.1,127.7,128.2,136.5$, 138.2, 148.7, 157.6, 167.5, 171.5, 173.4, 206.5. 118 
IR (KBr), $v\left(\mathrm{~cm}^{-1}\right): 3297,3058,2926,1701$, $1665,1560,1534,1438,1279,1222,1186$, $1063,836,743,559$.

\section{References}

[1] Kuroyanagi M, Fukuoka M, Yoshihira K, Natori S. Chemical and toxicological studies on bracken fern, Pteridium aquilinum var. latiusculum. III. Further characterization of pterosins and pterosides, sesquiterpenes and the glucosides having 1-indanone skeleton, from the rhizomes. Chem. Pharm. Bull. 1979; 27(3): 592-601.

[2] Finkielsztein LM, Alesso EN, Lanta B, Aguirre JM, Moltrasio GY. A simple synthetic route to pterosin F and other pterosins. J. Chem. Research (S) 1999; 406.

[3] Hsu FL, Huang CF, Chen YW, Yen YP, Wu CT, Uang BJ, Yang RS, Liu SH. Antidiabetic effects of pterosin A, a small-molecular-weight natural product, on diabetic mouse models. Diabetes. 2013; 62(2): 628-38.

[4] Yahara Y, Takemori H, Okada M, Kosai A, Yamashita A, Kobayashi T, Fujita K, Itoh Y, Nakamura M, Fuchino M, Kawahara N, Fukui N, Watanabe A, Kimura T, Tsumaki N. Pterosin B prevents chondrocyte hypertrophy and osteoarthritis in mice by inhibiting Sik. Nature Commun. 2016; 7: 10959.

[5] Bycroft BW, Payne DJ. Dictionary of Antibiotics and Related Substances. CRC Press; 2013, p. 1006.

[6] Okpekon T, Millot M, Champy P, Gleye C, Yolou S, Bories C, Loiseau P, Laurens A, Hocquemiller R. A novel 1-indanone isolated from Uvaria afzelii roots. Nat. Prod. Res. 2009; 23(10): 909-915.

[7] Rukachaisirikul V, Buadam S, Sukpondma Y, Phongpaichit S, Sakayaroj J, HutadilokTowatana $\mathrm{N}$. Indanone and mellein derivatives from the Garcinia-derived fungus Xylaria sp. PSU-G12. Phytochem. Lett. 2013; 6(1): 135-138.
[8] Nagle DG, Zhou Y-D, Park PU, Paul VJ, Rajbhandari I, Duncan CJG, Pasco DS. A New Indanone from the Marine Cyanobacterium Lyngbya majuscule That Inhibits Hypoxia-Induced Activation of the VEGF Promoter in Hep3B Cells. J. Nat. Prod. 2000; 63(10): 1431-1433.

[9] Ahmed N. Synthetic Advances in the Indane Natural Product Scaffolds as Drug Candidates: A Review. Studies in Natural Products Chemistry 2016; 51: 383-434.

[10] Patel VK, Dhrubo JS, Patel CN. Antimicrobial and antifungal screening of indanone acetic acid derivatives. J. Chem. Pharm. Res. 2010; 2(2): 50-56.

[11] Weinstock M, Bejar C, Wang RH, Poltyrev T, Gross A, Finberg JP, Youdim MB. TV3326, a novel neuroprotective drug with cholinesterase and monoamine oxidase inhibitory activities for the treatment of Alzheimer's disease. J. Neural. Transm. Suppl. 2000; (60): 157-69.

[12] Anderson GW, Zimmerman JE, Callahan FM. N-Hydroxysuccinimide Esters in Peptide Synthesis. J. Am. Chem. Soc. 1963; 85(19): 3039.

[13] Gershkovich AA, Kibirev VK. Chemical Synthesis of Peptides [in Russian]. Kyiv: Naukova Dumka; 1992, pp. 38-44.

[14] Shilin SV, Lenko IN, Shablykina OV, Khilya VP. 6-Oxyindan-1-ones with amino acid fragments. Fr. Ukr. J. Chem. 2018; 6(2): 18-26.

[15] Koelsch FC, Scheiderbauer RA. The Tautomerism of Indene. J. Am. Chem. Soc. 1943; 65(12): 2311-2314. 